\title{
Comparison of two methods of measurement of maximal respiratory pressures in health and cystic fibrosis
}

\author{
Theodore Dassios $^{1,2^{*}}$, Anna Katelari ${ }^{3}$, Stavros Doudounakis ${ }^{3}$, Gabriel Dimitriou ${ }^{2}$ \\ ${ }^{1}$ Cambridge University Hospitals NHS Foundation Trust, Cambridge, UK \\ ${ }^{2}$ Department of Paediatrics, University of Patras Medical School, Rio, Greece \\ ${ }^{3}$ Department of Cystic Fibrosis, Aghia Sophia Children's Hospital, Thivon \& Papadiamantopoulou, Athens, Greece \\ Email: " theodore.dassios@addenbrookes.nhs.uk, ${ }^{*}$ tdassios@upatras.gr
}

Received 19 June 2013; revised 26 July 2013; accepted 6 August 2013

Copyright (C) 2013 Theodore Dassios et al. This is an open access article distributed under the Creative Commons Attribution License, which permits unrestricted use, distribution, and reproduction in any medium, provided the original work is properly cited.

\begin{abstract}
Introduction: Respiratory muscle strength can be assessed by static mouth measurements of maximal inspiratory pressure $\left(\mathbf{P i}_{\max }\right)$ and maximal expiratory pressure $\left(\mathrm{Pe}_{\max }\right)$. Impaired respiratory muscle strength is common in neuromuscular and obstructive pulmonary disease such as Cystic Fibrosis (CF). Maximal respiratory pressures can easily be measured with a portable manometer on the bedside and in the community. Our objective was to compare maximal respiratory pressures as measured by standard laboratory equipment and the portable mouth pressure meter Micro RPM. Methods: $\mathbf{P i}_{\max }$ and $\mathrm{Pe}_{\max }$ were assessed in 296 healthy subjects and patients with CF with the Micro RPM and standard laboratory equipment. The Micro RPM measures and digitally displays maximal respiratory pressures after averageing over a one second period. Standard laboratory equipment consisted of a differential pressure transducer, whose amplified signals were analyzed by Lab-VIEW software. Each subject performed at least five reproducible maneuvers after familiarizing with the equipment. Results: The Micro RPM accurately measured maximal inspiratory and maximal expiratory pressures both in healthy individuals as well as in patients with CF. Mean difference (standard deviation) of the methods was $1.37(17.73) \mathrm{cm} \mathrm{H}_{2} \mathrm{O}$ for $\mathrm{Pi}_{\max }$ maneuvers and $1.84(9.09) \mathrm{cm} \mathrm{H}_{2} \mathrm{O}$ for $\mathrm{Pe}_{\max }$ maneuvers. Conclusions: The Micro RPM can reliably and accurately measure maximal respiratory mouth pressures and its use could be applied both in the clinical and the research setting.
\end{abstract}

Keywords: Respiratory Muscle Strength; Maximal Respiratory Pressures; Portable Manometry

"Corresponding author.

\section{INTRODUCTION}

Respiratory muscle strength testing is not routinely performed as part of the assessment of lung function in children or patients with Cystic Fibrosis (CF). Nevertheless, respiratory muscle weakness can lead to respiratory muscle fatigue and respiratory failure especially in individuals with compromised pulmonary reserves. Clinical assessment of respiratory muscle strength is difficult, thus justifying the need for objective quantitative measurements [1]. Respiratory muscle strength is compromised in COPD [2] and in conditions characterized by generalized muscular weakness [3], while malnutrition and steroid therapy are also known to negatively affect respiratory muscle strength [4]. Respiratory muscle impairment is also strongly associated with a negative impact on the clinical outcome of CF $[5,6]$. For all the aforementioned reasons, the physical therapist, clinician and health professional participating in the care of $\mathrm{CF}$ patients should be familiar with the technicalities of performing and the implications of interpreting respiratory muscle strength measurements.

The most widely used way to assess global respiretory muscle strength is by measuring static maximal inspiratory $\left(\mathrm{Pi}_{\max }\right)$ and static maximal expiratory $\left(\mathrm{Pe}_{\max }\right)$ pressures at the mouth [7]. Normal values for these indices have been established in both adult $[8,9]$ and pediatric populations [10]. A number of studies have reported decreased maximal respiratory pressure values in CF patients which are related to malnutrition and pulmonary function abnormalities [11,12]. Measurement of maximal respiratory muscle pressures has traditionally been carried out by complex devices in a research laboratory setting which demand the physical presence of the patient in the research lab. Handheld manometers allow for the physical therapist and clinician to test respiratory muscle strength both at the bedside as well as in the community. 
Thus, portable maximal respiratory pressure measuring needs to be accurate, reliable and easy to perform in a non-hospital setting.

Our objective was to compare measurements of static $\mathrm{Pi}_{\max }$ and $\mathrm{Pe}_{\max }$ pressures by a portable manometer against the standard lab equipment in a large cohort of healthy children and young adults as well as in children and young adults with $\mathrm{CF}$.

\section{METHODS}

\subsection{Study Design}

This study constituted part of a wider observational cross-sectional study investigating respiratory muscle function in patients with $\mathrm{CF}$.

\subsection{Subjects}

Patients with CF attending their follow up appointments in the Department of Cystic Fibrosis of Aghia-Sophia Children's Hospital in Athens, Greece and healthy children and young adults recruited in the outpatient department of Patras University Hospital, Patras, Greece were evaluated.

\subsection{Measurements}

The Micro RPM (Care Fusion, San Diego, California, USA) is a handheld manometer designed to measure maximal respiratory pressures which has been utilised to measure $\mathrm{Pi}_{\max }$ and $\mathrm{Pe}_{\max }[13,14]$ while it has also performed well in reliability testing both in the sitting and standing positions [15]. The inbuilt microprocessor is programmed to average the maximum pressure sustained over a period of one second both for inspiratory and expiratory maneuvers. Its features include measurement range of $+/-300 \mathrm{~cm} \mathrm{H}_{2} \mathrm{O}$, resolution of $1 \mathrm{~cm} \mathrm{H}_{2} \mathrm{O}$ and accuracy of $+/-3 \%$ [16].

The standard laboratory equipment consisted of a differential pressure transducer (DP45, range $\pm 225 \mathrm{~cm} \mathrm{H}_{2} \mathrm{O}$, Validyne Engineering, Northridge, California, USA), a carrier amplifier (Validyne CD 280, Validyne Engineering, Northridge, California, USA) and a computer (Dell Optiplex GX620, Dell Inc., Texas, USA) running data analysis software (Labview, National Instruments, Austin, Texas, USA) with analog-to-digital sampling at 100 Hz (16-bit NI PCI-6036E, National Instruments, Austin, Texas, USA). The standard laboratory equipment was calibrated before each subject against a handheld manometer. The maximum pressure sustained over one second was measured for both inspiratory and expiratory maneuvers.

$\mathrm{Pi}_{\max }$ was measured from Residual Volume (RV) performing a maximal inspiratory effort against an occluded airway and $\mathrm{Pe}_{\max }$ was measured from Total Lung Capac- ity (TLC) upon a maximal expiratory effort against an occluded airway [17]. Both maximal pressures were measured based on five maximal reproducible respiretory efforts and the maximum achieved value was recorded.

The respiratory line incorporated a small leak to avoid glottic closure [17]. Patients were all assessed with the same medical instruments in the same setting. Adequate time was allowed for the subjects to familiarize with the equipment and the technique. All participants were evaluated in the sitting position with a nose clip.

\subsection{Statistics}

Data were assessed for normality using KolmogorovSmirnoff and Shapiro-Wilk tests. Correlation testing was performed with Pearson Correlation Coefficient. Analysis of agreement between the two methods was performed according to the Bland and Altman recommendations [18]. Statistical analysis was performed using statistics software (SPSS 17.0, SPSS, Chicago, Illinois, USA).

\subsection{Ethics Approval}

The study protocol was approved by the Patras University Hospital and Aghia Sophia Children's Hospital Ethics Committees. Parents, legal guardians or patients as appropriate provided informed written consent prior to the study.

\section{RESULTS}

Between October 2009 and June 2010, 296 subjects were measured. 149 patients with CF and 147 healthy subjects were included. Ages ranged from 6 - 34 years. Median age was 13 years and interquartile range was $10-17$ years. In the CF group 70 patients were male (47\%), median age (interquartile range) was $14(10-17)$ years; while in the group of healthy subjects 85 of $147(58 \%)$ were male with a median age (interquartile range) of 13 $(10$ - 16) years.

In order to present the comparison between the two methods, the data obtained from healthy subjects and CF patients were pooled. However, the measured $\mathrm{Pi}_{\max }$ and $\mathrm{Pe}_{\max }$ data of both study subgroups are indicated by separate symbols in the corresponding figures.

Mean and standard deviation values for $\mathrm{Pi}_{\max }$ and $\mathrm{Pe}_{\max }$ by Micro RPM and Standard Laboratory (SL) methods are presented in Table 1. In the whole cohort there was a significant positive correlation between $\mathrm{Pi}_{\max }$ measured by Micro RPM and $\mathrm{Pi}_{\max }$ measured by $\mathrm{SL}(\mathrm{r}=$ $0.783, p<0.001$, Figure 1). In the whole cohort there was a significant positive correlation between $\mathrm{Pe}_{\max }$ measured by Micro RPM and $\mathrm{Pe}_{\max }$ measured by SL ( $\mathrm{r}=$ 0.953, $p<0.001$, Figure 2). In the CF group there was a 
significant positive correlation between $\mathrm{Pi}_{\text {max }}$ measured by Micro RPM and $\mathrm{Pi}_{\max }$ measured by SL $(\mathrm{r}=0.852, \mathrm{p}<$ $0.001)$ and between $\mathrm{Pe}_{\max }$ measured by Micro RPM and $\mathrm{Pe}_{\max }$ measured by SL $(\mathrm{r}=0.956, \mathrm{p}<0.001)$. In the healthy subjects there was significant positive correlation between $\mathrm{Pi}_{\max }$ measured by Micro RPM and $\mathrm{Pi}_{\max }$ measured by $\mathrm{SL}(\mathrm{r}=0.713, \mathrm{p}<0.001)$ and between $\mathrm{Pe}_{\max }$ measured by Micro RPM and $\mathrm{Pe}_{\max }$ measured by SL ( $\mathrm{r}=$ $0.951, \mathrm{p}<0.001)$. In children aged less than 12 years $(\mathrm{n}$ $=106$ ), there was a significant positive correlation of $\mathrm{Pi}_{\text {max }}$ measured by Micro RPM and $\mathrm{Pi}_{\text {max }}$ measured by SL $(\mathrm{r}=0.860, \mathrm{p}<0.001)$ as well as between $\mathrm{Pe}_{\max }$ measured by Micro RPM and $\mathrm{Pe}_{\max }$ measured by SL $(\mathrm{r}=0.940, \mathrm{p}<$ 0.001). Comparison of $\mathrm{Pi}_{\max }$ yielded a mean difference of $1.37 \mathrm{~cm} \mathrm{H} \mathrm{H}_{2} \mathrm{O}$ with a standard deviation of $17.73 \mathrm{~cm} \mathrm{H}_{2} \mathrm{O}$ (Figure 3). Comparison of $\mathrm{Pe}_{\max }$ showed a mean difference of $1.84 \mathrm{~cm} \mathrm{H}_{2} \mathrm{O}$ with a standard deviation of 9.09 $\mathrm{cm} \mathrm{H}_{2} \mathrm{O}$ (Figure 4).

\section{DISCUSSION}

Portable measurement of maximal respiratory pressures produces reliable and accurate results compared to the established standard laboratory technique. Comparison of the two methods yielded high correlation indices and a high level of agreement.
Maximal respiratory pressures are one of the most widely applied methods in the assessment of respiratory muscle strength; they carry the advantage that they are non-invasive, while a number of previous studies have established normal values in adults and children. Nevertheless, being volitional tests, one should consider that suboptimal motivation or lack of coordination might result in low readings in non-compromised, strong respiratory muscles [19]. In clinical practice, a measurement of maximal inspiratory pressure at $-80 \mathrm{~cm} \mathrm{H}_{2} \mathrm{O}$ practically excludes the possibility of respiratory muscle weakness [17].

The Micro RPM portable manometer offers the option to calibrate the device against a known reference static pressure. Over the nine month period of the study, we did

Table 1. Mean (SD) values of $\mathrm{Pi}_{\max }$ and $\mathrm{Pe}_{\max }$ as measured by the Micro RPM portable manometer and standard laboratory equipment (SL). Values are expressed in $\mathrm{cm}_{2} \mathrm{O}$.

\begin{tabular}{cc}
\hline $\mathrm{Pi}_{\max }$ Micro RPM & $72.6(27.1)$ \\
$\mathrm{Pi}_{\max } \mathrm{SL}$ & $73.8(26.7)$ \\
$\mathrm{Pe}_{\max } \mathrm{Micro} \mathrm{RPM}$ & $76.2(30.3)$ \\
$\mathrm{Pe}_{\max } \mathrm{SL}$ & $74.1(28.3)$ \\
\hline
\end{tabular}

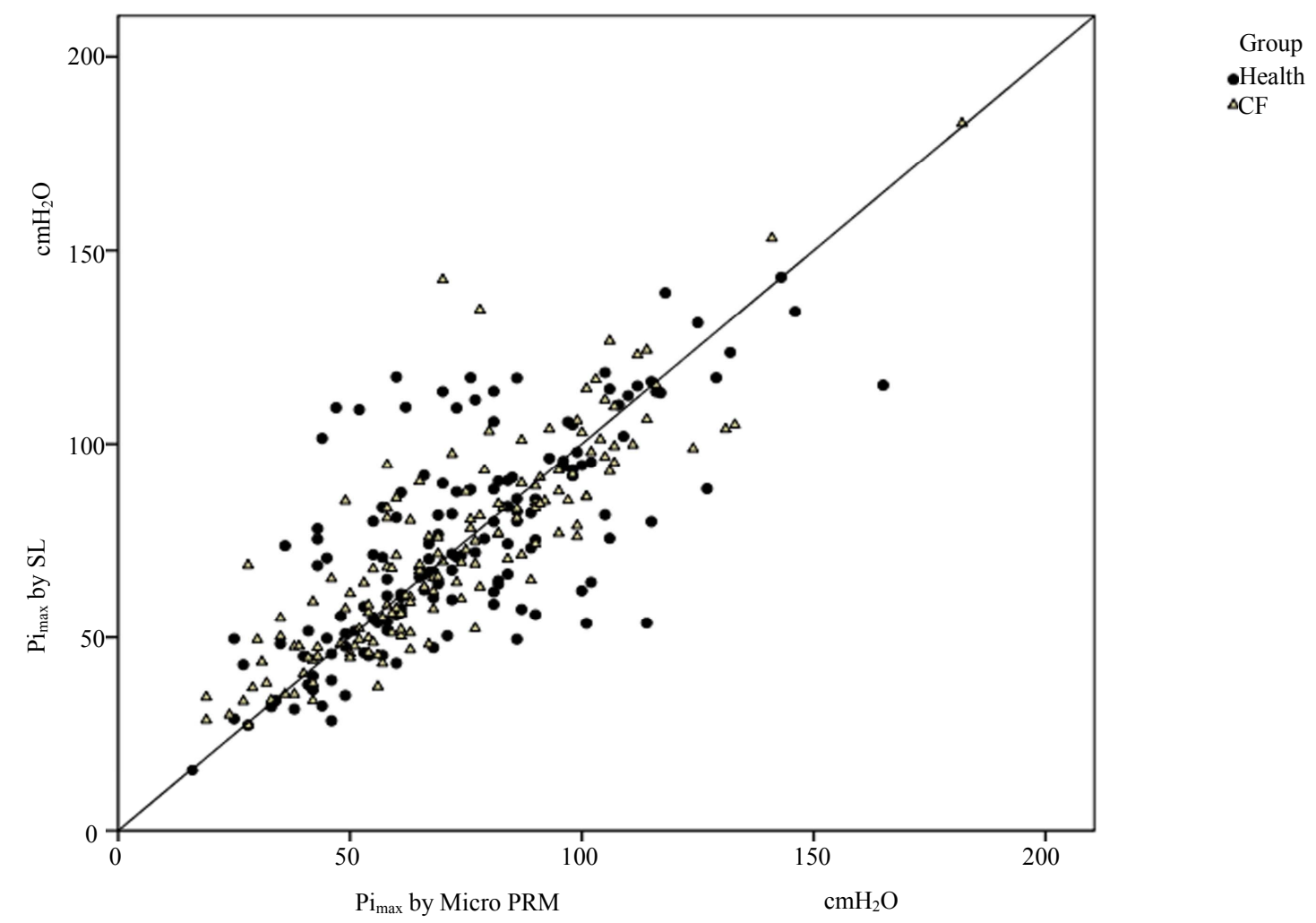

Figure 1. Comparison of maximal inspiratory pressure (Pimax) by Micro RPM portable manometer and by standard laboratory equipment (SL) with line of agreement. Data for CF patients and healthy subjects are indicated with separate symbols. 


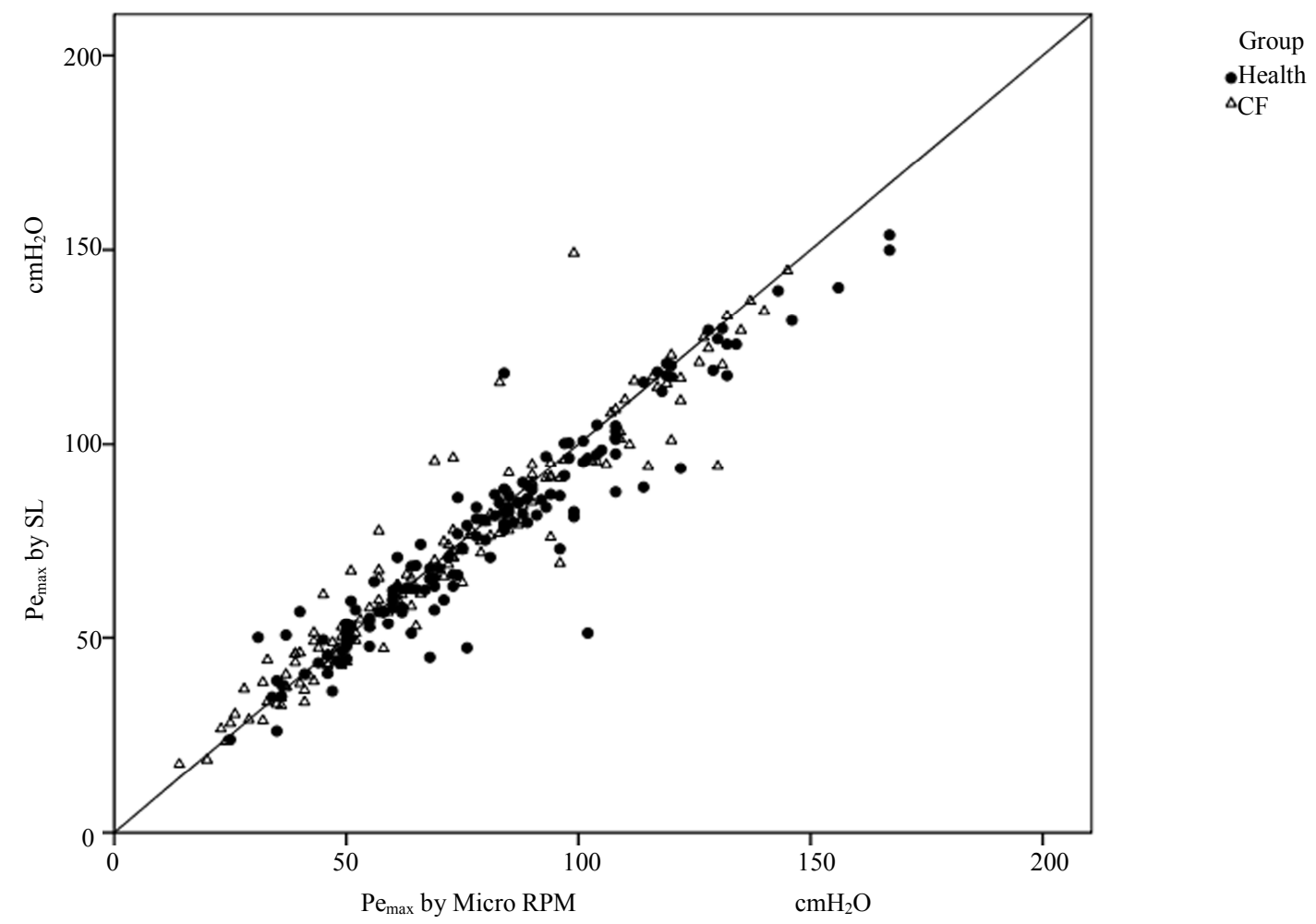

Figure 2. Comparison of maximal expiratory pressure (Pemax) by Micro RPM portable manometer and by standard laboratory equipment (SL) with line of agreement. Data for CF patients and healthy subjects are indicated with separate symbols.

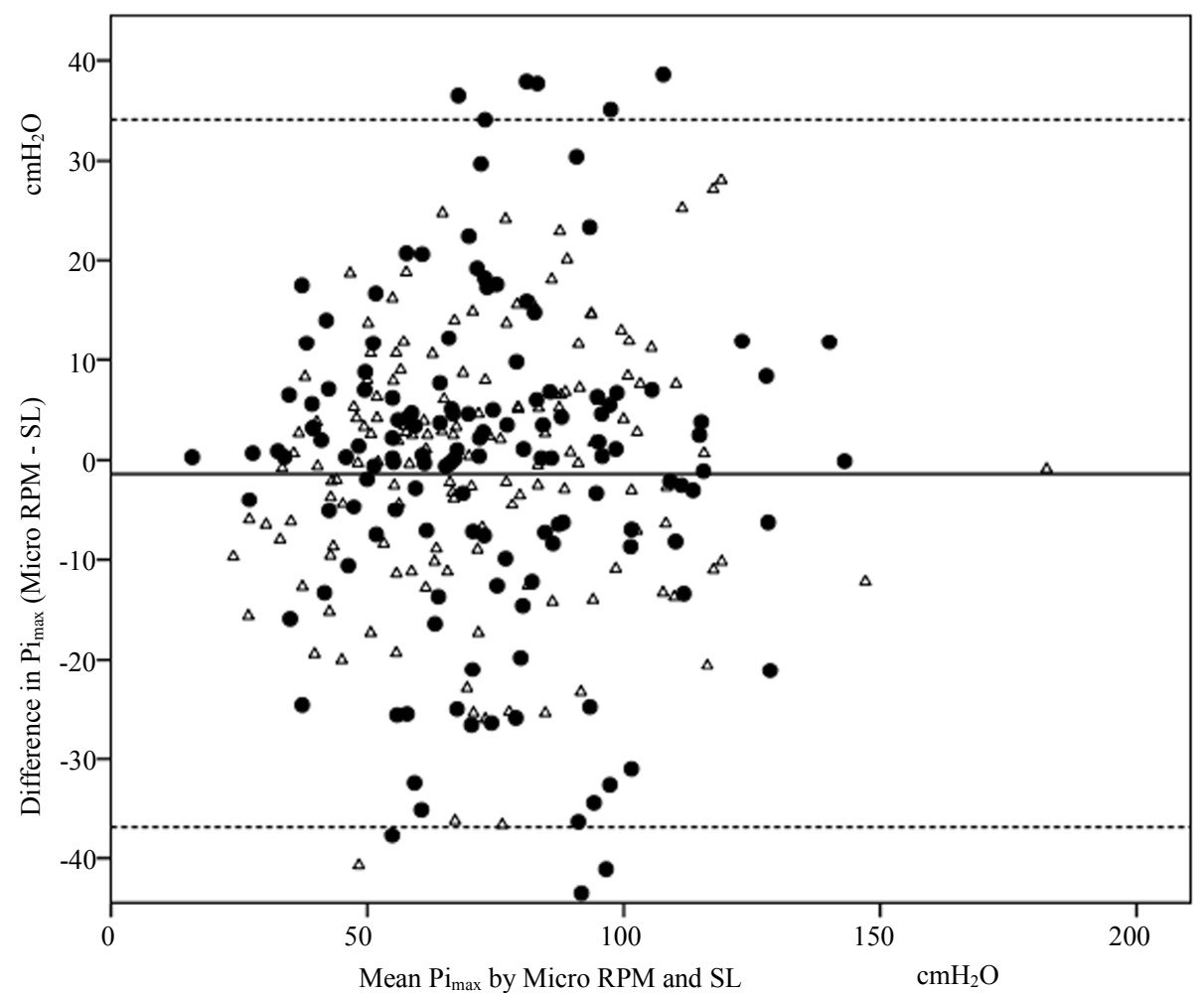

Group

-Health

$\triangle \mathrm{CF}$

Figure 3. Bland Altman plot of the difference between the two methods of measurement of Pimax over the mean values. Data for CF patients and healthy subjects are indicated with separate symbols. 


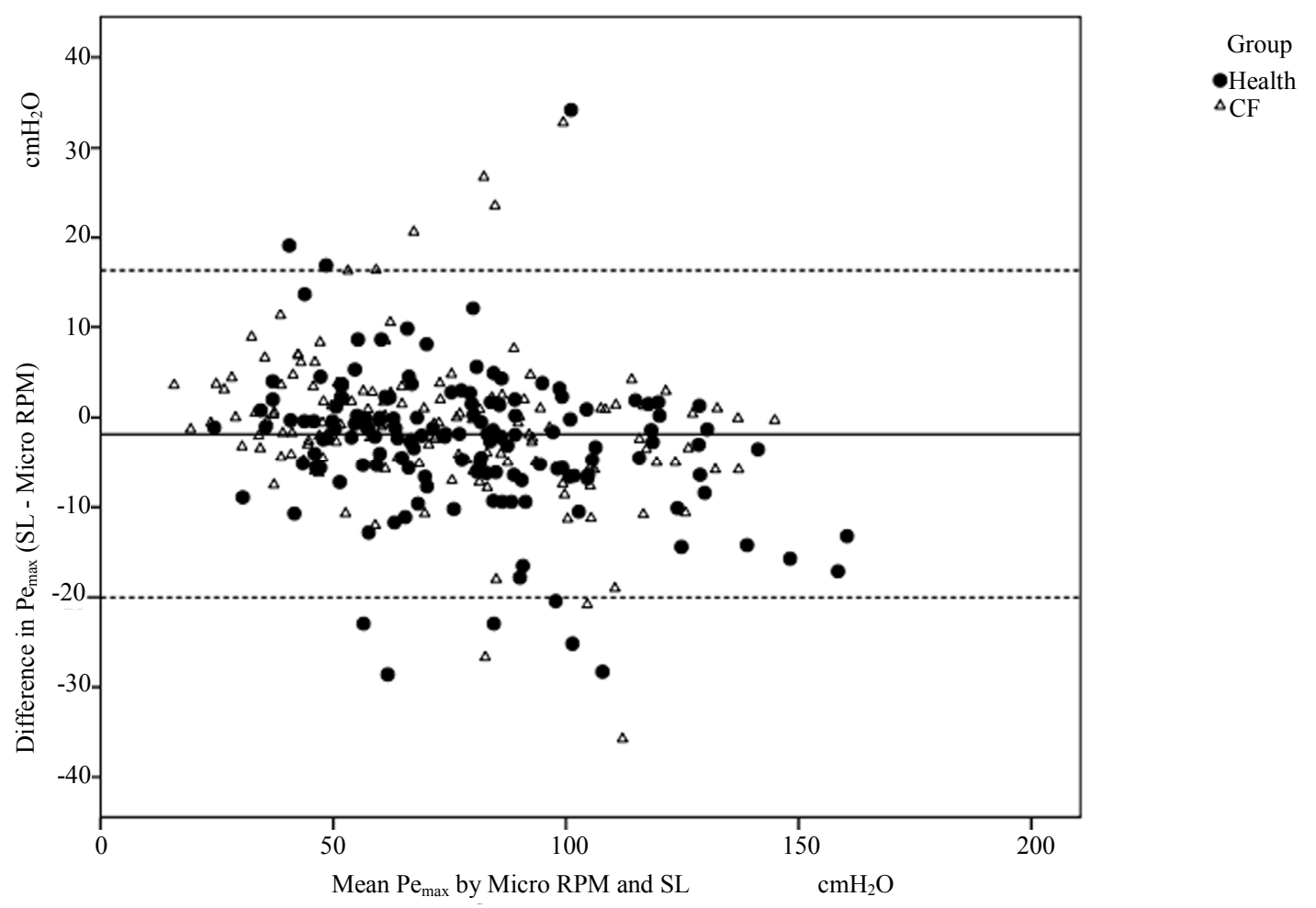

Figure 4. Bland Altman plot of the difference between the two methods of measurement of Pemax over the mean values. Data for CF patients and healthy subjects are indicated with separate symbols.

not perform static calibration of the Micro RPM and we did not observe any drift of measurements.

Estimation of maximal sustainable static pressure on Labview is traditionally performed by visual assessment of the pressure tracings and manual selection of a level at which the pressure is sustained at the plateau area of the curve; this might practically underestimate the actual averaged pressure over one second. The Micro RPM's feature of automatically processing and averaging pressure over the one second period might provide a more accurate estimation of the maximal pressure value.

The present study reports similar values for $\mathrm{Pi}_{\max }$ and $\mathrm{Pe}_{\max }$ in health and CF given that our cohort incorporates young children and adolescents $[11,12,20]$. $\mathrm{Pi}_{\max }$ measurements have a wider dispersion as compared to $\mathrm{Pe}_{\max }$ and as demonstrated in Table 1. This might be attributed to the increased technical difficulty of performing the maximal inspiratory maneuver and the unpleasant nature of the test, as compared to the maximal expiratory maneuver which is a rather more straightforward procedure. This unpleasant nature and need for motivation and coordination in performing the $\mathrm{Pi}_{\max }$ maneuver might be even more relevant in our study given that our cohort partly consists of young children.

The value of portable manometry in assessing respiratory muscle strength has been addressed in previous studies $[7,15,21]$. The present study differs to the previ- ous ones and complements them in that it consists of a large number of subjects and it incorporates young children and patients with Cystic Fibrosis. To the authors' best knowledge, no previous study has tested the device in young children where the anticipated maximal pressure values are lower [20], or in patients with CF where lung mechanics are distorted by obstruction and hyperinflation [22].

The authors believe that our findings bear significant importance both for clinical and research purposes. The Micro RPM is an easy to operate, portable, accurate and reliable device which could facilitate the spreading of respiratory muscle strength measurement in a variety of clinical environments, such as the hospital bedside and the community and assess the efficacy of physical therapy interventions such as inspiratory muscle training. Furthermore, this device could also be utilized in the research setting yielding accurate and reliable results. CF patients in particular could greatly benefit from regular measurement of $\mathrm{Pi}_{\max }$ and $\mathrm{Pe}_{\max }$, as they are known to be in increased danger or respiratory muscle fatigue and respiratory muscle failure $[23,24]$. Early recognition of these complications could help to decide when to initiate treatment modalities such as non-invasive ventilation and inspiratory muscle training.

The authors conclude that the Micro RPM reliably and accurately measures $\mathrm{Pi}_{\max }$ and $\mathrm{Pe}_{\max }$ both in healthy indi- 
viduals as well as in patients with $\mathrm{CF}$. The device is accessible and easy to operate and could help promote respiratory muscle strength assessment as a clinical and research tool.

\section{ACKNOWLEDGEMENTS}

The statistical guidance of Dr Richard Parker of the Centre for Applied Medical Statistics, University of Cambridge, UK is gratefully acknowledged.

\section{REFERENCES}

[1] Fauroux, B. and Lofaso, F. (2005) Measurements of respiratory muscle function in children. In: Hammer, J. and Eber, E., Eds, Paediatric Pulmonary Function Testing, Karger, Basel, 138. doi:10.1159/000083531

[2] Similowski, T. and Derenne, J.P. (1994) Inspiratory muscle testing in stable COPD patients. European Respiratory Journal, 7, 1871-1876. doi:10.1183/09031936.94.07101871

[3] Mulreany, L.T., Weiner, D.J., McDonough, J.M. et al. (2003) Noninvasive measurement of the tension-time index in children with neuromuscular disease. Journal of Applied Physiology, 95, 931-937.

[4] Barry, S.C. and Gallagher, C.G. (2003) Corticosteroids and skeletal muscle function in cystic fibrosis. Journal of Applied Physiology, 95, 1379-1384.

[5] Polkey, M.I., Kyroussis, D., Hamnegard, C.H., et al. (1996) Diaphragm strength in chronic obstructive pulmonary disease. American Journal of Respiratory and Critical Care Medicine, 154, 1310-1317. doi:10.1164/ajrccm.154.5.8912741

[6] Hart, N., Tounian, P., Clement, A., et al. (2004) Nutritional status is an important predictor of diaphragm strength in young patients with cystic fibrosis. The American Journal of Clinical Nutrition, 80, 1201-1206.

[7] Hamnegard, C.H., Wragg, S., Kyroussis, D., et al. (1994) Portable measurement of maximum mouth pressures. European Respiratory Journal, 7, 398-401. doi:10.1183/09031936.94.07020398

[8] Bruschi, C., Cerveri, I., Zoia, M.C., et al. (1992) Reference values of maximal respiratory mouth pressures: A population-based study. American Review of Respiratory Disease, 146, 790-793. doi:10.1164/ajrccm/146.3.790

[9] Black, L.F. and Hyatt, R.E. (1969) Maximal respiratory pressures: Normal values and relationship to age and sex. American Review of Respiratory Disease, 99, 696-702.

[10] Gaultier, C. and Zinman, R. (1983) Maximal static pressures in healthy children. Respiration Physiology, 51, 4561. doi:10.1016/0034-5687(83)90101-9

[11] Hayot, M., Guillaumont, S., Ramonatxo, M., et al. (1997) Determinants of the tension-time index of inspiratory muscles in children with cystic fibrosis. Pediatric Pulmonology, 23, 336-343.
doi:10.1002/(SICI)1099-0496(199705)23:5<336::AID-PP UL5>3.0.CO; 2-I

[12] Hahn, A., Ankermann, T., Claass, A., et al. (2008) Noninvasive tension time index in relation to severity of disease in children with cystic fibrosis. Pediatric Pulmonology, 43, 973-981. doi:10.1002/ppul.20887

[13] Soudon, P., Steens, M. and Toussaint, M. (2008) A comparison of invasive versus noninvasive full-time mechanical ventilation in Duchenne muscular dystrophy. Chronic Respiratory Disease, 5, 87-93. doi: $10.1177 / 1479972308088715$

[14] Klefbeck, B. and Hamrah Nedjad, J. (2003) Effect of inspiratory muscle training in patients with multiple sclerosis. Archives of Physical Medicine and Rehabilitation, 84, 994-999. doi:10.1016/S0003-9993(03)00133-3

[15] Dimitriadis, Z., Kapreli, E., Konstantinidou, I., et al. (2011) Test/retest reliability of maximum mouth pressure measurements with the MicroRPM in healthy volunteers. Respiratory Care, 56, 776-782. doi:10.4187/respcare.00783

[16] Carefusion (2010) Micro RPM, simple tests for respiretory muscle strength. Carefusion, Hoechberg.

[17] ATS/ERS (2002) ATS/ERS Statement on respiratory muscle testing. American Journal of Respiratory and Critical Care Medicine, 166, 518-624. doi:10.1164/rccm.166.4.518

[18] Bland, J.M. and Altman, D.G. (1986) Statistical methods for assessing agreement between two methods of clinical measurement. Lancet, 1, 307-310. doi:10.1016/S0140-6736(86)90837-8

[19] Syabbalo, N. (1998) Assessment of respiratory muscle function and strength. Postgraduate Medical Journal, 74, 208-215. doi:10.1136/pgmj.74.870.208

[20] Stefanutti, D. and Fitting, J.W. (1999) Sniff nasal inspiratory pressure. Reference values in Caucasian children. American Journal of Respiratory and Critical Care Medicine, 159, 107-111. doi:10.1164/ajrccm.159.1.9804052

[21] Severino, F.G., Resqueti, V.R., Bruno, S.S., et al. (2010) Comparison between a national and a foreign manovacuometer for nasal inspiratory pressure measurement. Revista Brasileira de Fisioterapia, 14, 426-431. doi:10.1590/S1413-35552010000500012

[22] Hart, N., Polkey, M.I., Clement, A., et al. (2002) Changes in pulmonary mechanics with increasing disease severity in children and young adults with cystic fibrosis. American Journal of Respiratory and Critical Care Medicine, 166, 61-66. doi:10.1164/rccm. 2112059

[23] Fauroux, B. (2011) Why, when and how to propose noninvasive ventilation in cystic fibrosis? Minerva Anestesiologica, 77, 1108-1114.

[24] Dassios, T., Katelari, A., Doudounakis, S., et al. (2012) Respiratory muscle function in patients with cystic fibrosis. Pediatric Pulmonology, in press. doi:10.1002/ppul.22709 